\title{
Le nouveau site du BMS est en ligne!
}

Clair, convivial, moderne et dynamique: voilà les attributs qui décrivent au mieux le nouveau site Internet du Bulletin des médecins suisses. Grâce à ses nouveaux outils, le site parvient non seulement à optimiser la présentation des informations sur le système de santé suisse, mais aussi à simplifier et à stimuler de manière attrayante l'échange d'opinions entre ses lecteurs.

Matthias Scholer

mscholer[at]emh.ch
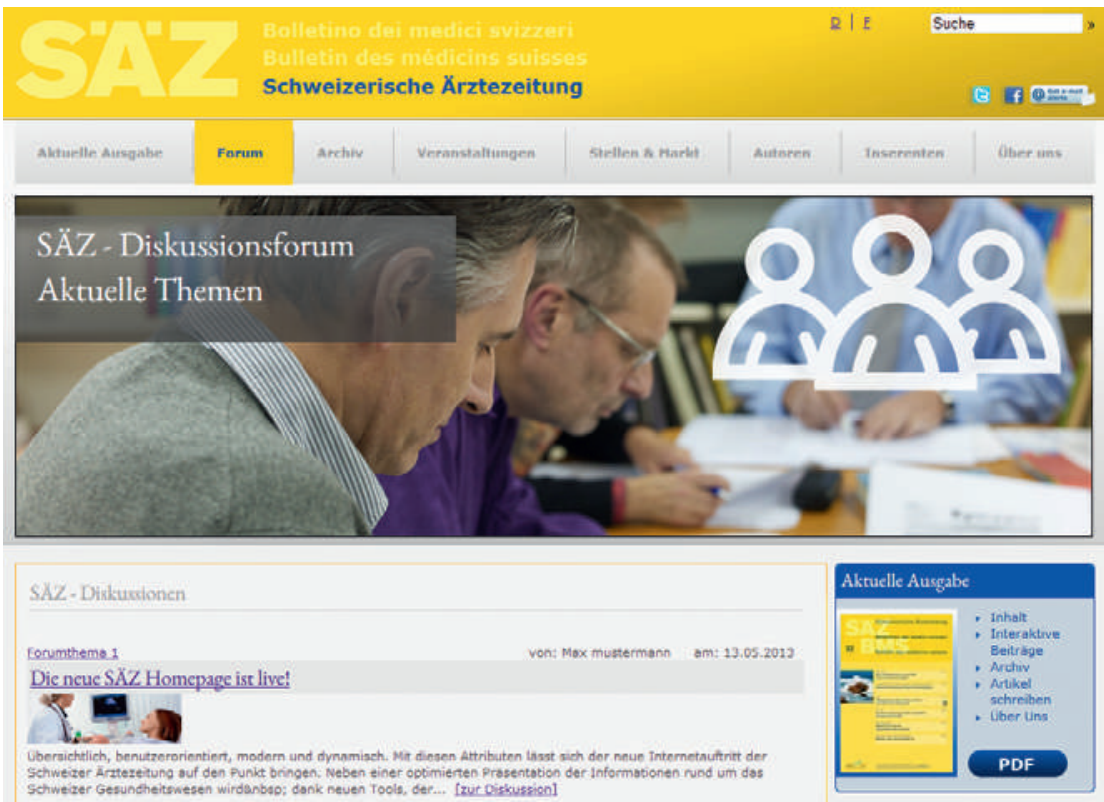

Figure 1

Le nouveau Forum permet une discussion interactive de sujets d'actualité (aussi en français).

\section{Articles interactifs

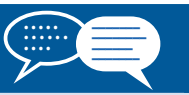 \\ Vous souhaitez commenter cet article? II vous suffit pour cela d'utiliser la fonction «Ajouter un commentaire» dans la version en ligne. Vous pouvez également consulter les remarques de vos confrères sous: www.bullmed.ch/ numero-actuel/articles-interactifs/}

\section{Figure 2}

Les utilisateurs peuvent commenter en ligne des articles sélectionnés.

Chacun de ces échanges est amorcé par une prise de position subjective aussi pointue que possible, dont l'auteur, homme ou femme, est soit une personnalité du monde politique ou économique, soit un acteur - médecin, infirmier ou patient - directement concerné par l'objet du débat. Les utilisateurs ont ensuite la possibilité de commenter l'article, de le compléter de leur avis personnel ou simplement d'approuver ou de rejeter un commentaire déjà publié à son sujet. Nous le faisons non seulement dans le but d'encourager l'échange actif d'opinions, mais aussi pour sonder l'humeur de notre lectorat sur les différents sujets.

De plus, des articles choisis de la version imprimée pourront à l'avenir être commentés en ligne. Ceux-ci sont signalés par un élément graphique spécial sur les pages du bulletin (fig. 2).

\section{Des prestations de service renforcées}

Est-ce la fin du courrier des lecteurs? Pas du tout. Dans la foulée de l'amélioration de l'offre de prestations en ligne, un masque de saisie a été spécialement créé pour les lettres de lecteurs (fig. 3 à la page suivante). En plus de faciliter le travail rédactionnel, celui-ci offre aussi aux auteur(e)s une possibilité commode de transmettre leurs textes à la rédaction. Le système leur signale l'éventuelle omission d'indications obligatoires et les aide à se conformer aux directives rédactionnelles. Ceci permet de réduire au strict minimum les échanges ultérieurs de courriels entre les auteurs et la rédaction et d'assurer ainsi une publication des lettres de lecteurs dans les meilleurs délais. 


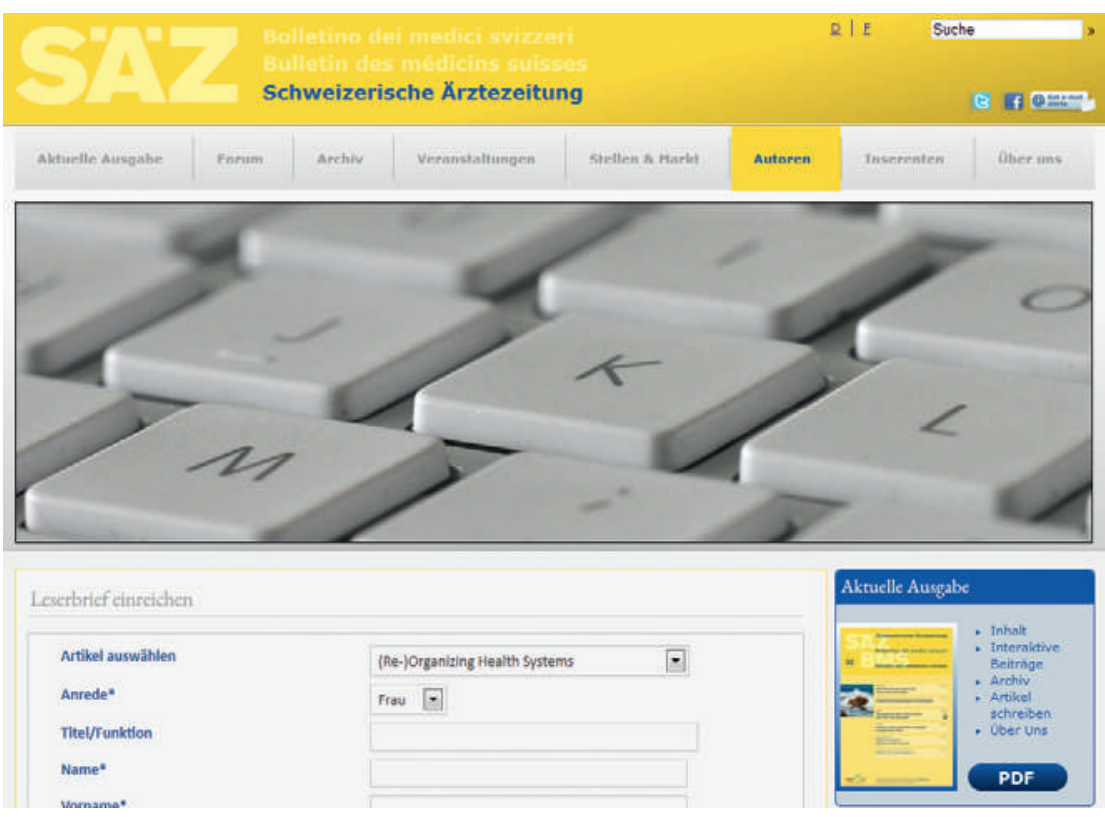

Figure 3

Le nouveau masque de saisie facilite l'envoi de lettres de lecteurs (aussi en français).

D'autres nouveautés destinées à améliorer la convivialité sont par exemple la procédure simplifiée d'insertion d'annonces publicitaires et événementielles ainsi que la possibilité pour les personnes en recherche d'emploi de mettre en ligne leur profil individuel.

\section{Actualité et multimédialité}

Afin de promouvoir le développement du site du BMS en une plate-forme d'information attrayante et moderne, une sélection d'articles et de nouvelles paraîtra dorénavant exclusivement en ligne. Ceci concerne notamment les sujets et les communiqués qui perdraient leur caractère d'actualité le temps qu'ils soient imprimés dans le bulletin. Ces informations seront dorénavant offertes en un mélange de différents formats (textes, vidéos et diaporamas commentés). Lorsque des productions sont impossibles ou trop coûteuses à réaliser en interne, les articles d'autres médias sont intégrés - ou cités avec référence aux sources - sur les pages de notre site. Ainsi, les utilisateurs peuvent avoir à tout moment, et en quelques clics de souris, un aperçu complet des nouvelles du système suisse de santé sans devoir rechercher laborieusement ces informations sur le réseau.

Chez EMH, nous sommes convaincus que ces adaptations constituent un pas important dans la bonne direction pour faire du Bulletin des médecins suisses, dans ses versions en ligne et imprimée, un organe de communication essentiel pour les médecins et appelé à durer. Dans cette perspective, nous nous faisons fort de respecter et d'honorer les habitudes et les besoins de nos fidèles lectrices et lecteurs.

De plus, celles et ceux qui trouvent la nouvelle présence enligne encore trop timide peuvent se réjouir de la venue prochaine d'une app BMS pour smartphones.

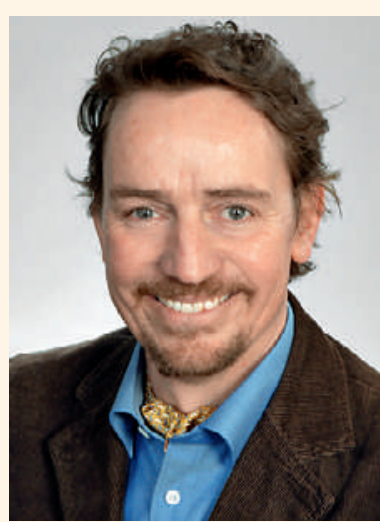

\section{Nouveau rédacteur de l'édition en ligne du BMS}

Matthias Scholer a dirigé la nouvelle conception du site Internet du BMS. Depuis ce mois de mai, il a entrepris d'en rédiger les contenus en étroite collaboration avec les autres membres de l'équipe rédactionnelle. Matthias Scholer a étudié la médecine vétérinaire à l'Université de Berne. Son doctorat en poche, il a travaillé dans la division de marketing d'une filiale de l'ancien «Institut suisse des sérums et vaccins». Après une formation en journalisme scientifique, Matthias Scholer a travaillé entre autres comme rédacteur à la «Fondation Maurice $\mathrm{E}$. Müller» et à la revue «Managed Care». Outre son activité chez EMH, il dirige les cours du CAS en journalisme spécialisé au Centre de formation médiatique de Lucerne (MAZ) et rédige régulièrement des articles scientifiques pour des médias grand public. 